\title{
Persuasion Language in Iranian Newspaper Ads
}

\author{
Foroogh kazemi
}

\author{
Assistant professor of Linguistics Department, Central Tehran Branch, Islamic Azad University, Tehran, Iran. \\ Email:f.kazemi86@yahoo.com \\ Talayeh Jafari \\ M. A Student of Linguistics, Central Tehran Branch, Islamic Azad University, Tehran, Iran. \\ Email: tjafari84@gmail.com
}

\begin{abstract}
This article has studied the language of persuasion in advertising texts and has examined persuasion in some Persian newspapers with an emphasis on the gender variable. This study was conducted by analytical approach and its data was collected from Iran's most important and widely circulated newspapers. The study results suggested that in persuasive texts of newspapers, the advertisers encourage viewers and audiences to buy the products by emphasizing on some characteristics specific to women such as beauty, adornment and housewifery as well as some special characteristics of men such as fitness, social prestige, responsibility and the role of being a provider. The findings indicated that the studied texts had characteristics in such a way that they use specific linguistic components and literary forms such as foregrounding, alliteration, hyperbole and exaggeration, metaphors and ambiguity to convey the desired concepts and attract the audience according to gender. Thus, the gender variable is an effective factor in the structure of persuasive texts related to advertising in Persian newspapers. The results of the research implied the fact that gender differences have been reflected in advertising in the form of language and other specific metalinguistic elements such as ideology and culture, and these linguistic and metalinguistic strategies are employed differently for men and women, in a way that this unequal gender approach seems to be man-centered.
\end{abstract}

Keywords: Advertising; Advertising texts of newspapers; Ideology; Persuasion; Persuasion techniques.

\section{1-Introduction}

Advertising is considered a kind of product supply and demand in the business world in which the supplier of the product tries to persuade the audience to buy and use the product in order to gain profit by presenting the product to the audience. On the other hand, language is and has always been a means of communication to satisfy human needs. Man expresses his desires, needs and emotions with language, is affected by it and affects others with it. Because language is a tool that can be used to affect others to achieve one's goals. Discourse analysis is a new method of research in communication texts that is used to understand the message and interpretation of communicative messages. This field of study focuses on the use of language in different contexts. One of the significant issues in the field of discourse analysis, and especially critical discourse analysis, is "persuasion". Persuasion is a part of discourse analysis and one of the most fundamental and practical aspects of communication which is used in many areas and means convincing, dominating and organizing. The current research is to explore the audience persuasion techniques in newspaper advertising and identify their features. The research is to find out which ideologies or linguistic and metalinguistic strategies the product advertisers use to persuade the audience in newspaper ads, and to discover whether these linguistic and metalinguistic strategies are used differently for men and women or not.

Several researches have been conducted by some researchers so far about advertising on television and radio and valuable results have been achieved. Because in these advertisements, image, characters, voice and speech are used to persuade the audience and perhaps the persuasion process is more effective in these cases. However, in newspaper advertising only text and pictures can be used. Therefore, studying the audience persuasion techniques in this case may include interesting results. In particular, this study has classified the advertisements according to the gender of the audience. The results of this study can be important for the providers of products and services to take advantage of various persuasion techniques and to better present the products to the audience on the one hand, and for the people, as consumers of those products and services, to decide better and easier when purchasing and using the products on the other hand. Therefore, it's a worthwhile topic to be addressed. In the present research, the data were collected by library method, and the methodology of the research was analytical. The data were collected from published ads in widely circulated newspapers, including Hamshahri, Iran, JameJam, Keyhan and Khabar Sport. The texts in these ads were classified based on male and female audiences, and they were analyzed according to audience persuasion techniques based on Hovland model (1949). For this purpose, all advertisements included in these newspapers were collected in ten days. These advertisements formed the corpus of the study.

\section{2- Review Of The Relevant Studies}

Kuhkan Kohangi (2012) has addressed the pragmatic analysis of the language of commercial advertisements (with a focus on subway ads). This research was conducted based on speech act theory. Its goal is to examine the language of commercial advertising and the role of indirect speech act in the effectiveness of advertisements and audience persuasion. Entezari Maleki (2014) has studied the induced functioning of the discourse system of advertising. This study has explored the discourse system of advertisements using a semiotics approach. Accordingly, he has classified some samples of different kinds of advertisements of billboards, magazines and media based on the desired discourse 


\section{ISSN $2348-3004$ \\ Volume $6 \mathrm{Number} 2$ \\ Journal of Advances in Linguistics}

functions. Geiss (1982) has studied the use of language in American television advertisements in his book. He focused not only on the language of advertising but also on the audience perception of the ads.

Vestergaard and Schroder (1985) have analyzed the language of commercial advertising in the press by using the communicative roles of language. They have considered the use of directive speech act to be important in persuading the audience to buy the products. From their point of view, commercial advertising can be a useful tool to apply different ideologies. In his book by the name of "The Art of Persuasion", Cheles (2001) has explored and analyzed the processes of expressing and transmitting political information and concepts in Italy after World War II. Alexander, Buehl and Mulhern (2002) have suggested in their article that some see persuasion as a negative word and an unfair interference in people. But that is not true, and this word has a positive meaning. Persuasion is the convincing of people to look differently and deeply to concepts and items. Abbas (2008) has studied the methods of persuasion used by Barack Obama in the 2008 president election. Grice (2010) has analyzed the persuasion methods used by three presidents of America in their lectures for children.

\section{3- Literature Review}

\section{3-1- The Process of Persuasion}

Persuasion means the convincing of people to have a tendency toward a particular topic and it is mainly a way of influencing and orienting the minds of specific individuals, especially the elites. It is a communication process that aims to influence the recipient of the message. A persuasive message presents an opinion or a behavior to the recipient voluntarily, which is expected to affect the audience or the recipient of the message. Persuasion is done through resorting to the attractionsinstead of using force and power. It is a process that often influences people's minds to change their behavior and compels them to do a certain action through using reasoning and emotion in the form of verbal, nonverbal and media skills. Although the process has the psychological property of freedom and the persuaded person feels that he's doing the determined goals and instructions by his own free will, it actually involves some sort of psychological pressure that resorts to emotions among logic and reasoning in the course of persuasion. In fact, persuasion is something between logic, reasoning, threats and punishment (Bingar, 1989: 10).

\section{3-1-1-Hovland persuasion model}

In 1949, Hovland, Janis and Kelly conducted a series of experiments in Yale University about changing attitudes. They used the findings in the formation of their theory on persuasion. They published their findings in 1953 in a book entitled "Communication and Persuasion". The persuasion model of Hovland et al. is the oldest model of convincing and changing attitudes. Based on Hovland's persuasion model, convincing is done through six fundamental steps as follows:

\section{Being exposed to the message}

If the intended audience does not hear or see the message, they won't be affected by it. Therefore, the first and fundamental condition of persuasion is for the audience to be exposed to the message.

\section{Paying attention to the message}

The second condition for the message to influence the audience is that they pay attention to the contents of the message.

3. Understanding the message

The audience needs to at least understand the conclusion of the message in order to be influenced by it.

4. Accepting the conclusion of message

The audience needs to accept the conclusion of the message in order to change attitude.

5. Retention of the new attitude

If the audience forgets the new attitude, the message will not be able to affect them in the future.

6. Converting the attitude to behavior

If the message affects the behavior, the new attitude can guide the behavior in position.

\section{3-2- Advertising}

Newspaper advertising is the methodical process of providing appropriate information about the products and services to customers and encouraging and persuading consumers to purchase goods and services effectively. It also involves a collection of profit methods in newspapers applied by financial institutions in order to attract, keep and increase buyers (Afshar Mohajer, 2007). In this area, the precise and scientific expression would be "Press Advertising". Press advertising is a subset of written advertisements.

\section{3-2-1- The relationship between persuasion and advertising}

"When the persuader hasn't gained many specific achievements in a persuasive relationship and his motivations are inconsistent with the interests of the other party, often the word of advertising is used, and the difference between them is minimal. Because both of them rely on communication tools to achieve predetermined goals" says Binglar (1989) about the relationship between persuasion and advertising.Tankard (2003) has defined persuasion as the manipulation of 
symbol to create action in others. In his opinion, persuasion and advertising are the same. The action or message can be called advertising only when one feels that the source of persuasion is not profiting, but the recipient of the message is.

\section{3-3- Gender}

One of the significant social factors causing linguistic diversity is gender. In describing gender, Crystal (1987) states that: the speech of men and women in languages such as Japanese, Thai and Caribbean have differences in pronunciation of grammatical and functional words. Different comments have been expressed about the main reason of the differences between the language behavior of men and women. Some believe that these differences result from various languages encountering and combining with each other and occur due to military, cultural, etc., conflicts. Others believe that the main reason for linguistic differences between men and women is the different social status of the two groups in society. Since women are generally expected to have a more appropriate social behavior, their manner of speech is socially better than men. Crystal has divided the reasons leading to differences between the speech of men and women into six categories: Biological, characteristic, cultural and role differences, male dominance and social gap between men and women.

\section{4- Data Analysis}

In this part of the research, the texts of newspapers advertisements were analyzed as follows:

\section{4-1- Advertisement of Kish twin towers (Jame Jam- July 25, 2015)}

This advertisement is allocated to the pre-sale of Kish twin towers. Since it involves some kind of trading and business, it's considered specifically as a masculine advertisement and deals with male audiences. In this advertisement, Kish commercial and residential towers are introduced, and the unique features of the twin towers are mentioned with phrases like "a window to nature, an experience of health, beyond expectation, a variety of sizes, minimum distance to the azure waters of the Persian Gulf, based on the world newest standards, health and recreational facilities". The ad tries to better show the towers' position in the region and the beach to the audience by presenting a number of pictures of the towers. The ad has used the color blue entirely for the background, text and the pictures of the towers, which somehow conforms perfectly to the sea and the blue sky and reflects the peace that will be waiting for the audience in these towers.

The "foregrounding" strategy has been used in this ad to persuade the audience. The presenter of the message has tried to draw the attention of the audience to the ad by introducing and foregrounding the features of the project with phrases like "a variety of sizes, minimum distance to the azure waters of the Persian Gulf, based on the world's newest standards, and health and recreational facilities". The "alliteration" technique has also been used in this ad. In this technique, it is attempted for the expressions used in the ad to rhyme like poems, so that they would be more convincing to the audience with their rhythmic nature. In the phrases "a window to nature" and "an experience of health", the two words "nature and health" rhyme (in Persian), which provides a harmonic and rhythmic structure in the text. The technique of "hyperbole and exaggeration" has also been used in this advertisement, which can be seen in the phrase "beyond expectation". This phrase implies a message to customers: these towers will be beyond your expectations with their unique situation and conditions, so don't hesitate to buy and enjoy their facilities, to have made a good investment, along with recreation and relaxation.

\section{4-2- West Computer Emergency advertisement (Hamshahri- July 23, 2015)}

This ad is dedicated to advertising of computer and laptops services. Since the computer is a tool mostly used in office and work environments and is nowadays an essential means of communication and managing administrative and business affairs, and since administrative and outdoor affairs are primarily the responsibility of men, so the audiences of this ad can mainly be considered the men of the society. In this ad, computer maintenance and repair services have been titled as "emergency". Also, in order for the audience to better understand the ad and the chosen name for the company providing services, a picture of a laptop along with a stethoscope, which is one of the necessary equipment in emergency rooms, has been displayed. An emergency room, in accordance with people's background information, is a place that provides immediate health services to people who are ill or have had an accident. The name "Computer Emergency" is also a right and proper choice for providing computer services of repair and maintenance, which means a place where immediate services are provided for damaged computers to regain their efficiency.

The technique of "metaphor" has been used in this ad to persuade the audience. Computer Emergency is a metaphor of hospital emergency. In computer emergency, the computers are repaired and their efficiency returned in the same way that people are treated and regain their health in hospital emergency rooms. The use of the phrase "least time, best services" emphasizes on providing better services in this company by using the "foregrounding" technique, where the computers are repaired in the shortest time and with the best quality. In this ad, the ideology describing this era as the age of speed and technology is conveyed to the audience, which implies the importance of speed and that it should not be wasted. Thus, the tools used in the workplace or at home should be repaired in the shortest time when damaged.

\section{4-3- Advertisement for Parsian Insurance Corp (Keyhan - August 1, 2015)}

This ad is allocated to Parsian Insurance. Since investing and thinking about the future and welfare of the family are the father's tasks, men can be considered as the particular audience of this advertisement. The ad displays a picture of a person sitting on a deck chair under an umbrella alongside a calm sea, completely relaxed. The umbrella over the person's head is a symbol of Parsian Insurance Corp logo, which shows that he has achieved peace by utilizing the services of Parsian Insurance and does not worry about his future any more, and the phrase "lots and lots of peace" which 
is the motto of Parsian Insurance emphasizes on that. This ad emphasizes on the quality of provided services to further draw the attention of the audience to the ad and to convince them with the use of the "foregrounding" technique and phrases like "provider of various insurances" and "recipient of the icon of excellence by the Consumer Protection Organization for respecting consumer rights". In this ad, the ideology of foresight, investment in affairs guaranteeing the future of the family, and supporting the family is conveyed to the male audience as the duties of men.

\section{4-4- Advertisement of Shahrak Furniture Repair (Keyhan - August 1, 2015)}

This ad is allocated to the repairing of furnishings. Since the furnishings are mostly used inside houses and most women are present in the home environment, women can be considered as the audience of this advertisement. The name of the service provider in this ad is "Shahrak", and in a way, the technique of "ambiguity" has been used in choosing the name of the service company. Since the company is located in "Shahrak Gharb" in Tehran, the name "Shahrak" is chosen for it. Hearing the name of the company evokes its location in the mind of the audience, and some kind of harmony can be found between them. Also, the use of the "foregrounding" technique and phrases such as "providing a variety of services, free transportation and visit, written warranty" emphasizes the distinguishing and outstanding features of the company and tries to persuade the audience and convince them to use the company's services by awareness of these features. This ad emphasizes on the ideology of women's housewifery and their presence at home, doing chores and taking care of affairs in the house and utilizing the modern services of urban life.

\section{4-5- Advertisement of Donyaye Omid Banquet Hall (JameJam - July 25, 2015)}

This ad introduces the services provided by Donyaye Omid banquet hall. Since holding wedding ceremonies and related matters mostly concern women, and women are particularly keen about holding wedding ceremonies, women are the main audience of this ad. The technique of "hyperbole and exaggeration" is used in choosing the name of this hall to persuade the audience. The hall is a small place in which various events take place. However, its name is chosen to be Donyaye Omid (The World of Hope) which is exaggerated and implies giving the costumer a world of hope. The hall motto -This place is the beginning of happiness- emphasizes more on the hope that it brings for the audience. The ideology conveyed to the female audience is that if she begins her life from this place, which is the beginning of happiness, she would live in a world full of hope. The advertisement continues to draw the attention of the audience to the outstanding features of the hall by introducing the facilities that distinguish it from other halls.

\section{4-6- Dessini advertisement (Jame Jam - July 24, 2015)}

This ad is dedicated to advertising Dessini products. Home appliances are the tools and equipment used at home, and women deal with them in home environments. Thus, according to Iranian tradition and culture, the main audience of this ad are women. This ad emphasizes on purchasing home appliances as the dowry of the bride, and implies that Dessini products can make the home environment more beautiful as a perfect and complete dowry. The motto of the products, "Home, Life, Dessini", has used the "metaphor" technique to persuade the audience. The metaphor is about the concept that the house will become a proper place to live with Dessini products. In this advertisement, a special gift is also prepared to further encourage the audience to buy the products. This ad conveys the ideology of consumerism to the audience.After some statistical analyses and studies are done, the table of differences of the persuasive texts of the press in Persian is presented as follows with regard to the issue of gender. This table indicates that the issue of gender has a direct effect on the selection of audience and the formation of the ad texts in Persian newspapers.

Table 1: The number and percentage of the studied newspaper ads classified by gender

\begin{tabular}{|c|c|c|c|}
\hline Row & Ads classification & Number & Percentage \\
\hline 1 & Ads with male audience & 153 & $77,27 \%$ \\
\hline 2 & Ads with female audience & 45 & $22,72 \%$ \\
\hline \multicolumn{2}{|c|}{ Total of the studied ads } & 198 & $100 \%$ \\
\hline
\end{tabular}

\section{5- Conclusion}

This study examined the language of persuasion in newspaper ads based on Hovland model. The findings indicated that specific linguistic forms such as foregrounding, metaphor, exaggeration, paronomasia and ambiguity, and metalinguistic forms such as ideology and culture have been used in the studied texts to persuade the audience to buy the product and convey the message. In these commercials, the audiences were persuaded to buy the products through motivating some gender qualities specific to women such as beauty, adornment, housewifery and modelling, and some special qualities of men such as fitness, gaining more acceptability in work environments and emphasizing on the providing role of men. The ideologies generally presented by advertising messages with female audience in the society focus mainly on paying attention to appearances and being comfortable when doing household chores. They lead the community of women toward a consumer society by presenting these concepts as important ones. The environment of these ads is mostly dedicated to the house and doing housework. The main pattern provided for women in these advertisements, is mainly about consumption, paying attention to small and insignificant details and life appearances, and deep humane concepts are not presented to women. In most newspaper ads, men are responsible for the management affairs of the house, and women and children play the role of obedient employees. These texts also emphasize on the tendency of men to use new 


\section{ISSN $2348-3004$ \\ Volume $6 \mathrm{Number} 2$ \\ Journal of Advances in Linguistics}

technologies, to avoid traditionalism and promote consumerism, to maintain a policy of masculinity in the house, and to maintain their social prestige and character to better perform outdoor social activities. The total number of ads specific to men was more than the ones specific to women, and one can say that these texts were mostly man-centered. In other words, the linguistic and metalinguistic strategies used in newspapers are different for men and women.

\section{REFERENCES}

[1] Afshar Mohajer, K. 2007. Press Graphics, Tehran, Samt Publications

[2] Entezari Maleki, S. 2014. Studying the induced functioning of the discourse system of advertis General linguistics master's thesis, Islamic Azad University, Central Tehran branch

[3] Binglar, A. 1989. Persuasive communications, Translation by Translators group of the research center,IRIB program studies and evaluation

[4] Tankard, J. 2003. Communication theories, Translation by Dehghan, A. Tehran University Publication, Tehran

[5] Kohkan Kohangi, S. 2012. Pragmatic analysis of the language of commercial advertisements (with a focuson subway ads). General linguistics master's thesis, Tarbiat Modarres University, Tehran

[6] Cheles, L. and Sponza, L. (ed). 2001. The Art of Persuation: Political Communication in Italy from 1945 to1900s. Manchester university press.

[7] Crystal, D. 1987. The Cambridge Encyclopedia of Language, 2nd edition, Cambridge University Press.

[8] Fakhruddin, A. 2008. The Use of Persuasive Technique by Barack Obama in His Political Speeches in the 2008 USA Presidential Primaries. The State Islamic University of Malang.

[9] Geis, M. L. 1982. The Language of Television Advertising. London: Academic Press.

[10] Grice, P. 2010. Presidential Communication to Children: An Analysis of Persuasive Strategies Presidential Speeches. Masters Theses \& Specialist Projects. Paper 185.

[11] Hovland, C., I. Lumsdaine, Sheffield, F. D. 1949. Experiments on mass communication. PrincetPrinceton University Press.

[12] Patricia , A. \& Fives, H. \& Buehl , M. \& Mulhern, J. 2002. "Teaching as Teacher Education". Vo118 , p.795.

[13] Vestergaard, T. , Schroder, K. 1985. The Language of Advertising. Oxford: Blackwell.

Biography of the first author: Foroogh Kazemi is assistant professor of linguistics department in Central Tehran Branch, Islamic Azad University in Iran. She has taught linguistics, Persian and English at Central Tehran Branch, Imam Khomeini International University and Science and Research branch. Dr. Kazemi has published many articles in linguistics and has been advisor, reader and referee of many linguistic MA theses and judge of articles in journal of linguistics. Her areas of interest are functional linguistics, sociolinguistics, typology and discourse analysis. She is the head of linguistics department in Central Tehran Branch, Islamic Azad University.

Biography of the second author: Talayeh Jafari was born in Tehran in 1984. She is BS of Physical Education at Central Tehran Branch, Islamic Azad University, in 2007. She is now, MA student of linguistics, at Central Tehran Branch, Islamic Azad University. 